\title{
Models for Infrared and Submillimetre Counts and Backgrounds
}

\author{
Michael Rowan-Robinson \\ Astrophysics Group, Blackett Laboratory, Imperial College of Science \\ Technology and Medicine, Prince Consort Road, London SW7 $2 B Z$
}

\begin{abstract}
A simple and versatile parametrized approach to the star formation history allows a quantitative investigation of the constraints from far infrared and submillimetre counts and background intensity measurements.

The models include four spectral components: infrared cirrus, an M 82-like starburst, an Arp 220-like starburst and an AGN dust torus. The $60 \mu \mathrm{m}$ luminosity function is determined for each chosen rate of evolution using the $\mathrm{PSCz}$ redshift data for 15000 galaxies. The proportions of each spectral type as a function of $60 \mu \mathrm{m}$ luminosity are chosen for consistency with IRAS and SCUBA colour-luminosity relations, and with the fraction of AGN as a function of luminosity found in $12 \mu \mathrm{m}$ samples.

A good fit to the observed counts at $0.44,2.2,15,60,90,175$ and $850 \mu \mathrm{m}$ can be found with pure luminosity evolution in all 3 cosmological models investigated: $\Omega_{o}=1, \Omega_{o}=0.3(\Lambda=0)$, and $\Omega_{o}=0.3, \Lambda=0.7$. All 3 models also give an acceptable fit to the integrated background spectrum. The total mass-density of stars generated in all 3 cosmological models is consistent with that observed.
\end{abstract}

\section{Introduction}

Surveys at $850 \mu \mathrm{m}$ (Hughes et al. 1998; Eales et al. 1999; Barger et al. 1999; Blain et al. 2000; Fox et al. 2000) and the detection of the submillimetre background (Puget et al. 1996; Fixsen et al. 1998; Hauser et al. 1998) have opened up highredshift dusty star-forming galaxies to view. Here I review what source counts and the background radiation at infrared and submillimetre wavelengths can tell us about the star formation history of the universe. Madau et al. (1996) showed how the ultraviolet surveys of Lilly et al. (1996) could be combined with information on UV dropout galaxies in the Hubble Deep Field to give an estimate of the star formation history from $z=0-4$. However these studies ignored what is already well-known from far infrared wavelengths, that dust plays a major role in redistributing the energy radiated at visible and UV wavelengths to the far infrared.

Subsequent to the Madau et al. analysis, several groups of authors have argued that the role of dust is crucial in estimates of the star formation rates at high redshifts. Rowan-Robinson et al. (1997) derived a surprisingly high rate of star formation at $z=0.5-1$ from an ISO survey of the Hubble Deep Field 

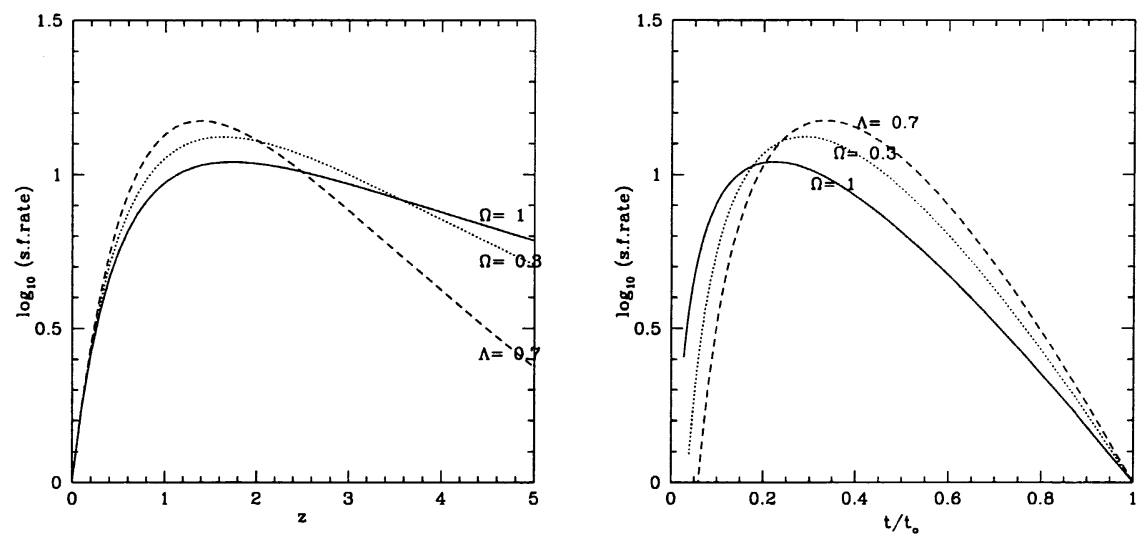

Figure 1. (Left) Star formation histories of the form of Eq. (1), as a function of $z$, from best-fit models for far infrared and submillimetre counts and background for $\Omega_{o}=1$, [solid curve, $(P, Q)=(1.2,5.4)$ ]; $\Omega_{o}=0.3$, [dotted curve, $\left.(P, Q)=2.1,7.3\right)$ ]; $\Lambda=0.7$, [broken curve, $(P, Q)=(3.0,9.0)]$.

(Right) Same, but as a function of cosmic time, $t$.

(HDF). Subsequent ISO estimates by Flores et al. (1999) confirmed the need to correct for the effects of dust in estimates of star-formation rates. Large extinction correction factors (5-10) have also been derived at $z=2-5$ by Meurer et al. (1997, 1999), Pettini et al. (1998) and Steidel et al. (1999).

Here I report the results of a parametrized approach to the star formation history of the universe, which allows a large category of possible histories to be explored and quantified. The parametrized models can be compared with a wide range of source-count and background data at far infrared and submillimetre wavelengths to narrow down the parameter space that the star formation history can occupy. The approach is similar to that of Blain et al. (1998) and Guiderdoni et al. (1998), but differs in key respects outlined below. The models of Franceschini et al. (1997) invoke a new population of heavily obscured high redshift starbursts, designed to account for the formation of spiral bulges and ellipticals, whereas I am testing whether the submillimetre background and counts can be understood in terms of a single population of evolving star-forming galaxies. A full account is given by Rowan-Robinson (2000, ApJ submitted).

A Hubble constant of $100 \mathrm{~km} \mathrm{~s}^{-1} \mathrm{Mpc}^{-1}$ is used throughout.

\section{Parametrized Approach to Star Formation History}

In this paper I present a parametrized approach to the problem, investigating a wide range of possible star formation histories for consistency with counts and background data from the ultraviolet to the submillimetre.

The constraints we have on the star formation rate, $\dot{\phi}_{*}(t)$, are that

(i) it is zero for $t=0$ 

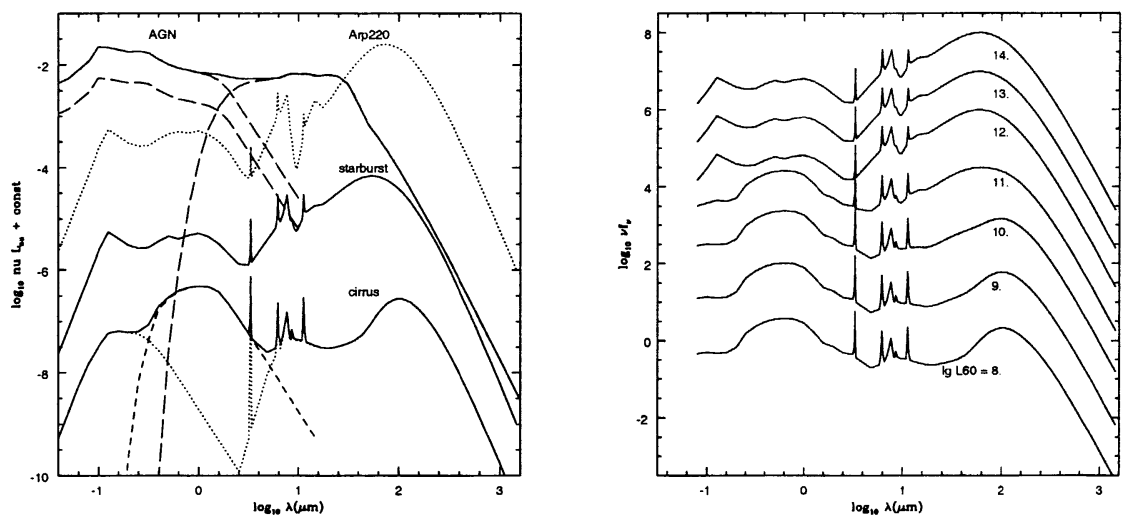

Figure 2. (Left) Adopted spectral energy distributions for the four components adopted in this study: cirrus [with optical emission split into low-mass (broken curve) and high-mass (dotted curve) stars]; M 82-starburst; Arp 220-starburst (models from Efstathiou et al. 2000); AGN dust torus (model from Rowan-Robinson 1995), showing assumed optical/IR ratio at $\log _{10}\left(L_{60} / L_{\odot}\right)=14$ (upper curve) and 8 .

(Right) Average SED as a function of $60 \mu \mathrm{m}$ luminosity, ranging from $\log _{10}\left(L_{60} / L_{\odot}\right)=8$ to 14 .

(ii) it is finite at $t=t_{o}$

(iii) it increases with $z$ out to at least $z=1$ (and from (i) must eventually decrease at high $z$ ).

A simple mathematical form consistent with these constraints is

$$
\dot{\phi}_{*}(t) / \dot{\phi}_{*}\left(t_{o}\right)=\exp \left\{Q\left[1-\left(t / t_{o}\right)\right]\right\}\left(t / t_{o}\right)^{P}
$$

where $P$ and $Q$ are parameters $[P>0$ to satisfy (i), $Q>0$ to satisfy (iii)]. I assume that $\dot{\phi}_{*}(t)=0$ for $z>10$.

Equation (1) provides a simple but versatile parametrization of the star formation history, capable of reproducing most physically realistic, single-population scenarios. Figure 1 shows models of type Eq. (1) derived from fits to far infrared and submillimetre source-counts and background intensity in different cosmological models (see below). Models of the form (1) will not, however, be able to reproduce the very sharply peaked scenario ('ED') of Dwek et al. (1998), or the two-population model of Franceschini et al. (1997). In the scenarios modelled by Eq. (1) the formation of bulges and ellipticals are part of an evolving spectrum of star-formation rates.

The physical meaning of the parameters is as follows. The exponential term describes an exponential decay in the star formation rate on a time-scale $t_{o} / Q$, which can be interpreted as a product of the process of exhaustion of available gas for star formation (a competition between formation into stars and return of gas to the interstellar medium from stars) and of the declining rate of galaxy 
interactions and mergers at later epochs. This parameter is the same as that used in the galaxy SED models of Bruzual and Charlot (1993). The power-law term represents the build-up of the rate of star formation due to mergers of fragments which are incorporated into galaxies. $P$ measures how steeply this process occurs with time. The ratio $P / Q$ determines the location of the peak in the star formation rate, $t_{\text {peak }}$, since $t_{\text {peak }} / t_{o}=P / Q$.

A very important assumption in the present work is that the star formation rate should vary smoothly with epoch. Several earlier assumptions have assumed, for mathematical convenience, discontinuous changes of slope in the star formation rate (e.g., Pearson \& Rowan-Robinson 1996; the Blain et al. 1998 'anvil' models). Such discontinuities are highly unphysical and I have eliminated them in this work.

We might expect that the cosmological model could have a significant effect on the relationship between predicted counts and predicted background intensity, since the latter is sensitive to how the volume element and look-back time change with redshift. To test this, I have explored models with (a) $\Lambda=0$, for which all the required formulae are analytic (specifically, here, models with $\Omega_{o}=1$, 0.3 ); and (b) $k=0$, for which some of them are analytic (specifically, $\Lambda=0.7$, $\left.\Omega_{o}=0.3\right)$.

\section{3. $60 \mu \mathrm{m}$ Luminosity Function and Evolution Derived from the IRAS PSCz Survey}

Given an assumed $(P, Q)$, I then determine the $60 \mu \mathrm{m}$ luminosity function, using the IRAS PSCz sample (Saunders et al. 1999). I fit this with the form assumed by Saunders et al. (1990)

$$
\eta(L)=C_{*}\left(L / L_{*}\right)^{1-\alpha} \exp \left\{-0.5\left[\log _{10}\left(1+L / L_{*}\right) / \sigma\right]^{2}\right\}
$$

It is not clear that any previous studies have correctly taken account of the need to change the $60 \mu \mathrm{m}$ luminosity function as the rate of evolution is varied. The study of Guiderdoni et al. (1998) explicitly violates the known constraints on the $60 \mu \mathrm{m}$ luminosity function at the high luminosity end, and as a result the models predict far too many high redshift galaxies at a flux-limit of $0.2 \mathrm{Jy}$ at $60 \mu \mathrm{m}$, where substantial redshift surveys have already taken place. Blain et al. (1998) state that they have determined the luminosity function from consistency with the $60 \mu \mathrm{m}$ counts, but this process does not automatically give detailed consistency with existing redshift surveys.

We can also use the PSCz data to determine a range of consistency for $(P, Q)$, using the $V / V_{m}$ test. The predicted uncertainty in $\left\langle V / V_{m}\right\rangle$ for a population of $n$ galaxies, $(12 n)^{-1 / 2}$, can be used to assign a goodness of fit for each set of $(P, Q)$ values.

\section{Assumed Infrared and Submillimetre Spectral Energy Distribu- tions}

To transform this $60 \mu \mathrm{m}$ luminosity function to other wavelengths, we have to make an assumption about the spectral energy distributions. I have explored a 
variety of assumptions about these SEDs: (a) a single SED at all luminosities representative of starbursts; (b) composite SEDs which are a mixture of four components, a starburst component, a 'cirrus' component, an Arp 220-like starburst, and an AGN dust torus (Rowan-Robinson 1995), with the proportions of each depending on $60 \mu \mathrm{m}$ luminosity. Neither of these approaches gave satisfactory results, and it was not possible to find a simultaneous fit to all the far infrared and submillimetre counts and background spectrum in any cosmological model. Finally, I have derived counts and background spectrum separately for each of the four components and then summed. This approach finally gave satisfactory fits to all available data. It also allows a correct determination of the redshift distribution of each type separately as a function of wavelength and flux-density, and the proportion of each type of contribution to the counts at any flux-density or to the background.

I have used the latest predictions for infrared SEDs of these components by Efstathiou et al. (2000), and added near IR-optical-UV SEDs corresponding to an Sab galaxy for the cirrus and an HII galaxy for the starburst component, respectively. The starburst model is a good fit to multiwavelength data for M 82 and NGC 6092 (Efstathiou et al. 2000), and also to far infrared and submillimetre data for luminous starbursts (Rigopoulou et al. 1996).

The normalization between far infrared and optical-UV components is determined by $L(60 \mu \mathrm{m}) / L(0.8 \mu \mathrm{m})=0.15$ for the cirrus component, 5.3 for the starburst component, and 49 for the Arp 220 component. For the AGN component, I assume that $L(10 \mu \mathrm{m}) / L(0.1 \mu \mathrm{m})=0.3$ (cf Rowan-Robinson 1995) for the most luminous AGN, and that this ratio increases with decreasing luminosity to account for the fact that the mean covering factor is higher at lower luminosities. So $\log \{L(10 \mu \mathrm{m}) / L(0.1 \mu \mathrm{m})\}=-0.52+0.1 \times(14.0-\log L 60)$.

For the cirrus component I have, somewhat arbitrarily, divided the optical SED into a contribution of young, high-mass stars $(\lambda \leq 0.4 \mu \mathrm{m})$ and a contribution of old, low-mass stars $(\lambda \geq 0.4 \mu \mathrm{m})$ (see Fig. 2). The former are assumed to trace the star formation rate, but the latter trace the cumulative star formation up to the epoch observed. This treatment, though approximate, allows a reasonable prediction of the $K$ - and $B$-band counts. The two components in Fig. 2 can be modelled, assuming $L \propto M^{3}$, blackbody SEDs with $T \propto L^{1 / 2}$, and with a Salpeter mass function, with mass-range $0.1-1 M_{\odot}$ for the low-mass star component, and 8-40 $M_{\odot}$ for the high-mass star component.

The proportions of the four components (at $60 \mu \mathrm{m}$ ) as a function of luminosity, $t_{i}\left(L_{60}\right)$, have been chosen to give the correct mean relations in the $S(25) / S 12), S(60) / S(25), S(100) / S(60)$ and $S(60) / S(850)$ versus $L(60)$ diagrams. Where predictions are being compared with IRAS $12 \mu \mathrm{m}$ data, or (later) with ISO $15 \mu \mathrm{m}$ or $6.7 \mu \mathrm{m}$ counts, account is taken of the width of the relevant observation bands by filtering with a top-hat filter of appropriate half-width $(0.23,0.26$ and 0.16 at $6.7,12$, and $15 \mu \mathrm{m}$ respectively). Otherwise, observations were assumed to be monochromatic. The relative proportion of the $60 \mu \mathrm{m}$ emission due to AGN dust tori as a function of $L(60)$ is derived from the luminosity functions given by Rush et al. (1993) (see Fig. 4). The resulting mean SEDs as a function of $L(60)$ are shown in Fig. 2. Luminosity functions at different wavelengths are shown in Figs. 3-4. There is good agreement with measured luminosity functions at wavelengths from $0.44-850 \mu \mathrm{m}$. The fit to the $850 \mu \mathrm{m}$ 

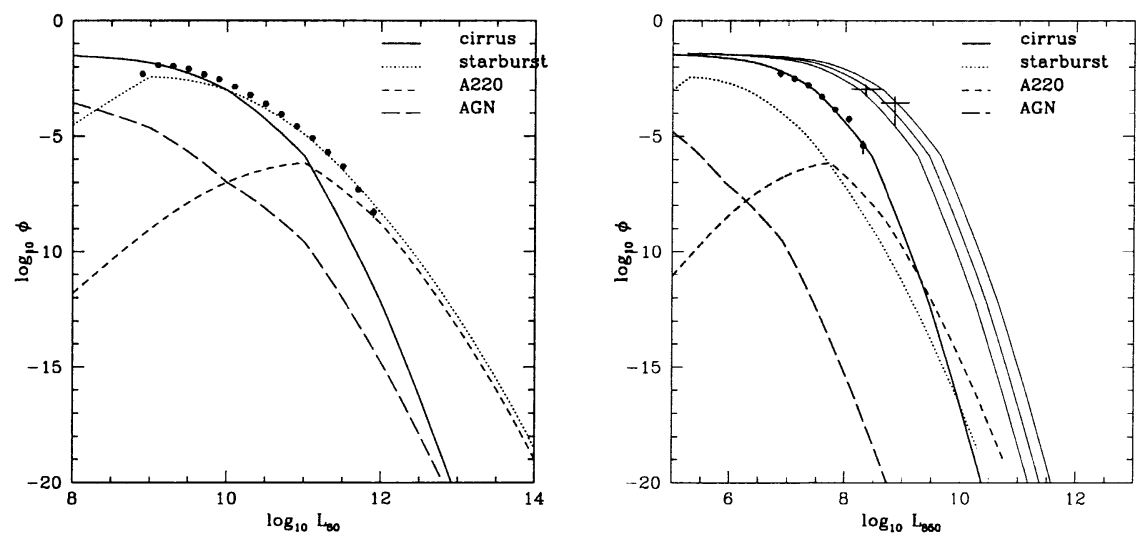

Figure 3. (Left) Luminosity functions at $60 \mu \mathrm{m}$ for the 4 spectral components. Units of $\phi$ are $\mathrm{Mpc}^{-3} \mathrm{dex}^{-1}$, luminosity $\left(\nu L_{\nu}\right)$ in solar units. All luminosity functions are for $\Omega_{o}=1$ model, $H_{o}=$ $100 \mathrm{~km} \mathrm{~s}^{-1} \mathrm{Mpc}^{-1}$. Observed points are derived from $\mathrm{PSCz}$ data.

(Right) Luminosity functions at $850 \mu \mathrm{m}$ for the 4 spectral components. The filled circles are data from Dunne et al. (2000). The crosses are derived from the data of Hughes et al. (1998) for the HDF.

luminosity function of Dunne et al. (2000) is impressive, since the transformation from 60 to $850 \mu \mathrm{m}$ is based only on choosing the $t_{i}\left(L_{60}\right)$ to give the correct average $S(100) / S(60)$ as a function of $L_{60}$. It is also impressive that luminosity functions derived in the far infrared can fit the data at $0.44 \mu \mathrm{m}$ ( $B$ band): the only freedom in the models to fit the $B$-band luminosity functions and counts is the amplitude of the optical SED relative to the far infrared.

Clearly it will be important to have submillimetre data for a wide range of normal and active galaxies to test and improve these SEDs. But the approach of using accurate radiative transfer models, with realistic assumptions about dust grains, which have been verified with observations of known galaxies, seems superior to modelling the SED as a blackbody with power-law dust grain opacity in which the dust temperature is treated as a free parameter (as in Blain et al. 1998). The latter approximation can only be valid for rest-frame wavelengths greater than $60 \mu \mathrm{m}$, i.e., for accurate prediction of counts and background intensities at wavelengths $>200 \mu \mathrm{m}$. Useful predictions can certainly not be made at $15 \mu \mathrm{m}$ without explicit treatment of PAHs. These criticisms do not apply to the studies of Guiderdoni et al. (1998), Dwek et al. (1998), Xu et al. (1998) and Dole et al. (2000), whose assumed SEDs are similar to those used here.

I have also assumed that the same luminosity evolution function should be applied to the whole $60 \mu \mathrm{m}$ function, i.e., to AGN, 'cirrus' and 'starburst' components. I have investigated the effect of making the switchover in proportions of different types of component at a fixed luminosity, so that there is in effect a strong increase in the proportion of galaxies that are starbursts (or contain AGN) with redshift. However, this did not permit a fit to all the available data. 

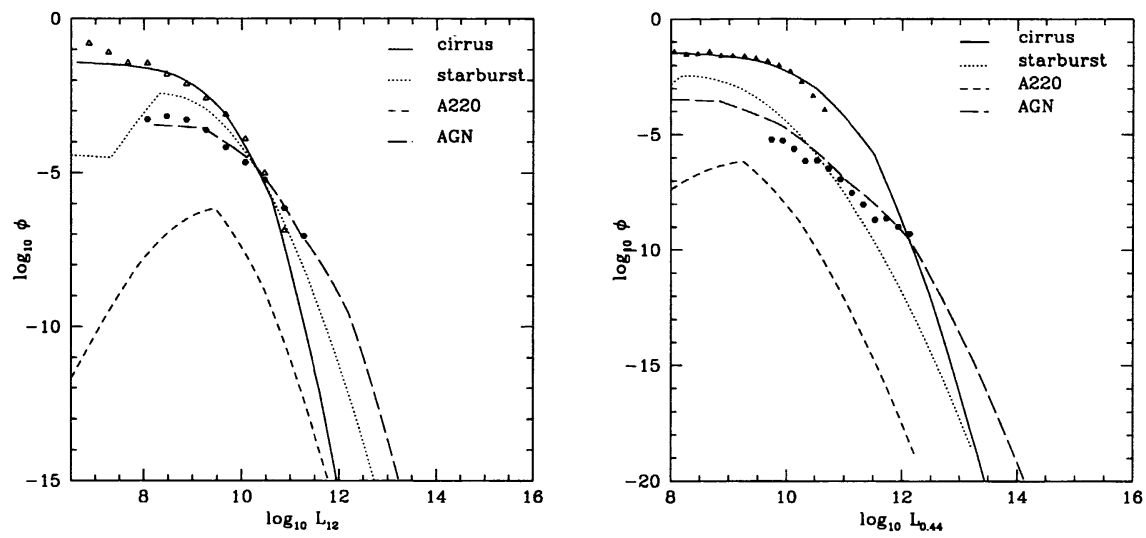

Figure 4. (Left) Luminosity functions at $12 \mu \mathrm{m}$ for the 4 spectral components. Observed points taken from Rush et al. (1993) (filled circles: Seyferts, open triangles: non-Seyferts).

(Right) Luminosity functions at $0.44 \mu \mathrm{m}$ for the 4 spectral components. Data for quasars derived from PG sample and for galaxies from Loveday et al. (1992).

A substantial part of the illumination of the cirrus component in spiral galaxies is due to recently formed massive stars, part of whose light escapes directly from star-forming regions despite the high average optical depth in these regions. In the starburst models of Efstathiou et al. (2000), this corresponds to the late stages of their starburst models. If the typical starburst luminosity was greater in the past, then the emission from interstellar dust in the galaxy would also be correspondingly greater. I have not at this stage considered the evolution of the SEDs of each component with redshift (see discussion in section 8).

It is possible that the evolution of AGNs differs from that of starbursts at $z>3$, but this will have little effect on the far infrared counts and background (there could be a significant effect at $15 \mu \mathrm{m}$, which will be worth further study).

Elliptical galaxies are not treated explicitly, though their star formation rate must have been much greater in the past than at present. I am assuming that ellipticals are quiescent starburst galaxies, that their star formation proceeded in much the same way as we see in current live starbursts, and that their star formation episodes are part of the evolution history quantified here. We have to think of this history as a series of short-lived fireworks taking place in different galaxies at different times. Similarly this approach does not track the different spiral types separately, but only in a global average at each epoch.

\section{Combined Fits to 60,175 and $850 \mu \mathrm{m}$ Counts, and 140-750 $\mu \mathrm{m}$ Background, and Determination of $P, Q$}

I can now predict the counts and background intensity at any wavelength, and, by comparing with observed values, constrain loci in the $P-Q$ plane. To 
determine $(P, Q)$ for any given cosmological model, I combine the constraints found at $60 \mu \mathrm{m}$ from the PSCz (section 4 above) with constraints from (1) deep counts at $60 \mu \mathrm{m}(50 \mathrm{mJy}),(2)$ the observed source-counts at $850 \mu \mathrm{m}$ at 1 and $4 \mathrm{mJy}$, and (3) the background intensity at 140,350 and $750 \mu \mathrm{m}$. For all 3 cosmological models, values of $(P, Q)$ can be found which give a satisfactory fit to all the available data. As emphasized above, this outcome does depend strongly on the assumptions made about the SEDs.

An important constraint on the models is that the total mass of stars produced in galaxies should be consistent with the mass of stars observed, $\Omega_{*}=0.003 \pm 0.0009 h^{-1}$ (Lanzetta et al. 1996), and that it should be less than the total density of baryons in the universe, $\Omega_{*} \leq 0.0125 \pm 0.0025 h^{-2}$ (Walker et al. 1991).

We can calculate the total mass-density of stars from the $60 \mu \mathrm{m}$ luminosity density using Eq. (7) of Rowan-Robinson et al. (1997), modified to take account of the latest Bruzual and Charlot galaxy evolution models (Rowan-Robinson 2000), from

$$
\Omega_{*}=10^{-11.13} \xi h^{-2} l_{60}\left(t_{o} / 10 \mathrm{Gyr}\right)
$$

where $l_{60}$ is the luminosity density in solar luminosities per $\mathrm{Mpc}^{3}$,

$$
\xi=\int_{0}^{1}\left[\phi_{*}(t) / \phi_{*}\left(t_{o}\right)\right] d\left(t / t_{o}\right),
$$

and the fraction of optical-UV light being radiated in the far infrared has been assumed to be $\epsilon=2 / 3$.

The models which fit the counts and background for $\Omega_{o}=1(P=1.2$, $Q=5.4), \Omega_{o}=0.3(P=2.1, Q=7.3)$, and $\Lambda=0.7(P=3.0, Q=9.0)$ give $l_{60}=4.3,4.4$ and $4.1 \times 10^{7} h \mathrm{~L}_{\odot} \mathrm{Mpc}^{-3}$ respectively, and $\xi=5.70,6.66$, 7.25 , so the corresponding values of $\Omega_{*}$ are $0.0027,0.0032$ and $0.0033 h^{-1}$, for $t_{o}=13 \mathrm{Gyr}$, consistent with observations. Estimating this from the young stellar component at $2800 \AA$ or from the $K$-band luminosity density (with an assumed mass-to-light ratio) also gives consistent results for an assumed Salpeter mass-function.

\section{Predicted Counts and Integrated Background Spectrum from the UV to the Submillimetre}

Figures $5-8$ show the predicted source-counts in the 3 selected cosmological models at $850,175,90,60,15,2.2$ and $0.44 \mu \mathrm{m}$. The agreement with observations at infrared and submillimetre wavelengths is extremely impressive. Although the fits at 850 and $60 \mu \mathrm{m}$ have been ensured by the least-squares procedure for determining $P$ and $Q$, the fits at 175,90 and $15 \mu \mathrm{m}$ are simply a consequence of the assumed SEDs and the choice of the $t_{i}\left(L_{60}\right)$. There is not much difference between the predictions of the 3 cosmological models at $60-850 \mu \mathrm{m}$. At $15 \mu \mathrm{m}$ there is a difference between the models in the predicted numbers of sources at fluxes below $100 \mu \mathrm{Jy}$. The proportion of AGN dust tori at $12 \mu \mathrm{m}$ agrees well with the data of Rush et al. (1993) (15\% brighter than 0.4 Jy). Fig. 7 shows that the proportion of AGN at $15 \mu \mathrm{m}$ is reasonably constant (15-20\%) for fluxes brighter than $3 \mathrm{mJy}$, but is predicted to fall rapidly towards fainter fluxes. 

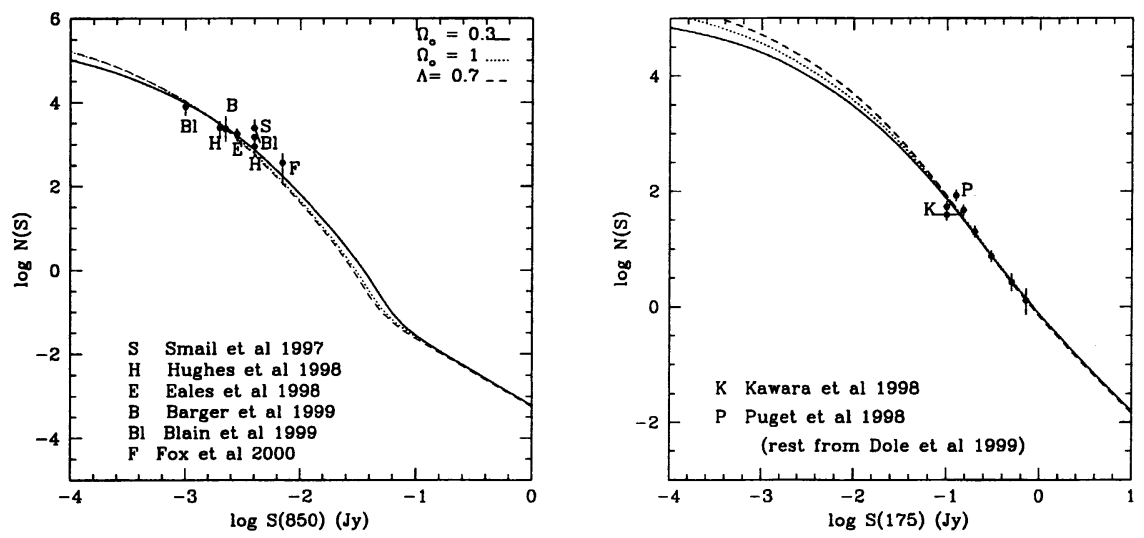

Figure 5. (Left) Integral source counts at $850 \mu \mathrm{m}$. Data are from Hughes et al. (1998), Eales et al. (1999), Smail et al. (1997), Barger et al. (1999), Blain et al. (2000), and Fox et al. (2000). The three models shown are, from bottom at faint fluxes, for $\Omega_{o}=1$ and $(P, Q)=(1.2$, 5.4), (solid curve); for $\Omega_{o}=0.3,(P, Q)=(2.1,7.3)$ (dotted curve); and for $\Lambda=0.7,(P, Q)=(3.0,9.0)$ (broken curve).

(Right) Source counts at $175 \mu \mathrm{m}$. Data points are from Kawara et al. (1998), Guiderdoni et al. (1998), and Dole et al. (2000).
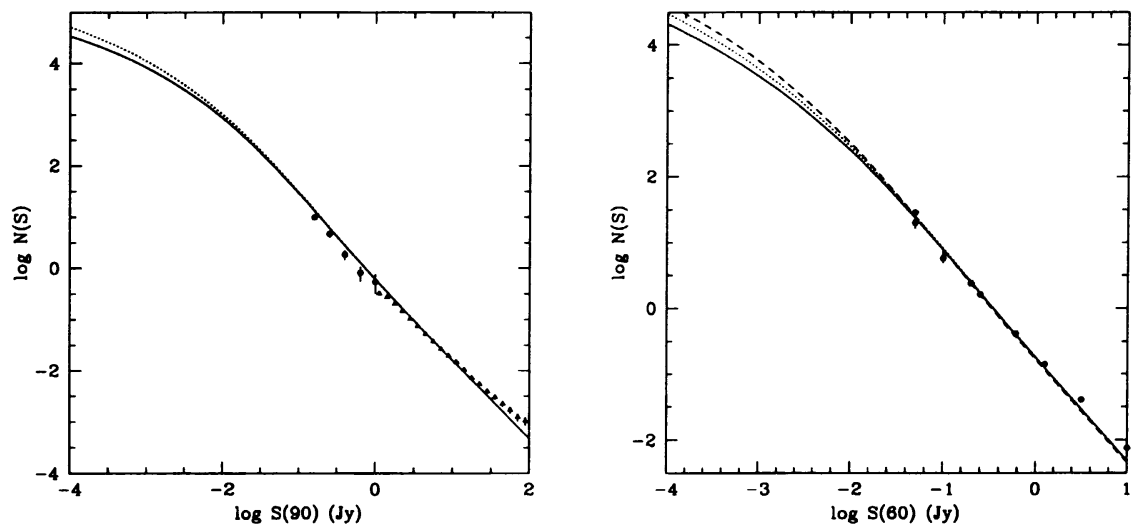

Figure 6. (Left) Source-counts at $90 \mu \mathrm{m}$. Data from IRAS PSCz (triangles) and ELAIS (filled circles) are from Efstathiou et al. (2000). (Right) Source counts at $60 \mu \mathrm{m}$. Data are from Lonsdale et al. (1990) (at 0.2-10 Jy), Hacking \& Houck (1987) (at 50-100 mJy), RowanRobinson et al. (1991), and Gregorich et al. (1995) (higher point at $50 \mathrm{mJy}$ ). Models as in Fig. 5. 


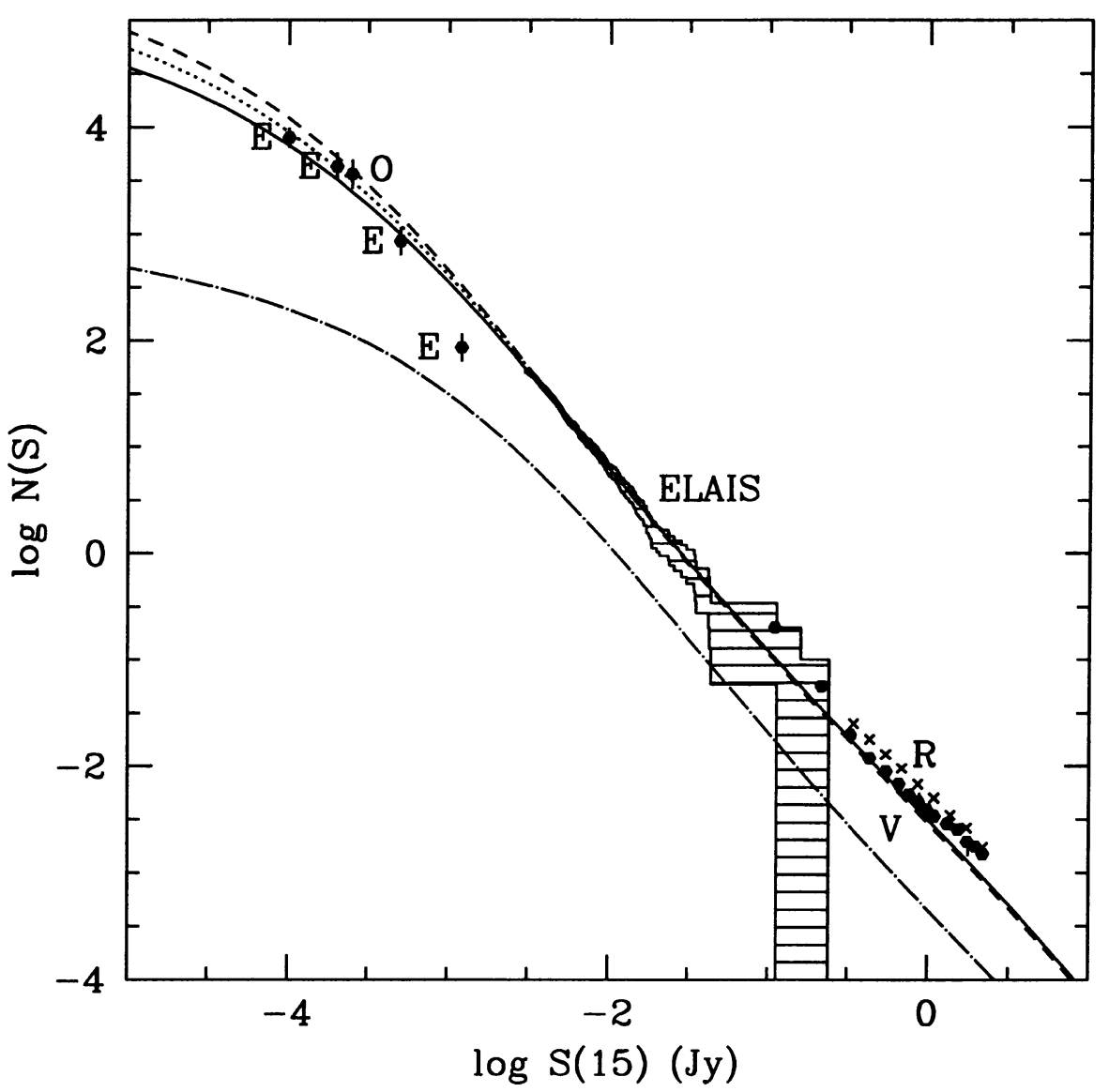

Figure 7. Source counts at $15 \mu \mathrm{m}$. Data from Oliver et al. (1997, O), Serjeant et al. (2000, ELAIS), Elbaz et al. (2000, E) Rush et al. (1993, R, crosses), Verma (2000, V, filled circles). Models as in Fig. 5. The lower dash-dotted line shows counts of the AGN dust torus population for the $\Omega_{o}=1$ model. 

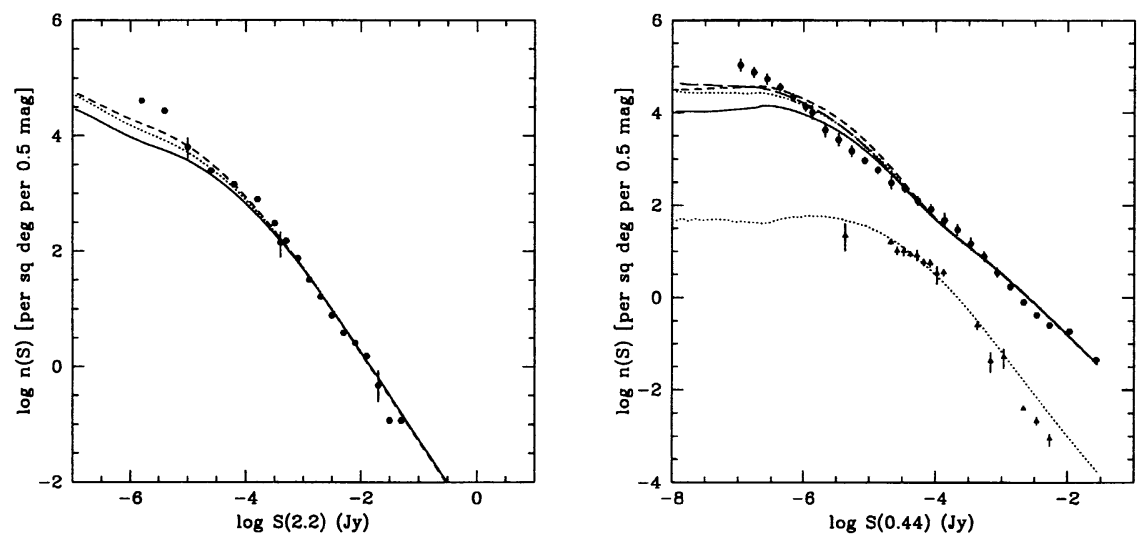

Figure 8. (Left) Differential source counts at $2.2 \mu \mathrm{m}$. Data from McCracken et al. (2000).

(Right) Differential source counts at $0.44 \mu \mathrm{m}$. Galaxy data (filled circles) are from Metcalfe et al. (1995), quasar data (triangles) from Boyle et al. (1988). Models as in Fig. 5. The long-dashed line shows the effects of including density evolution (see text) on the $\Omega_{o}=1$ model.

The model does not at present have ingredients capable of accounting for the very faint $K$ - and $B$-band galaxy counts. This could be provided either by a measure of density evolution (see below) or by steepening the faint-end luminosity function at $z>1$, which could in either case be attributed to a population of galaxies that had merged into present-day galaxies.

Figure 9 shows redshift distributions at selected wavelengths and fluxes. The median redshift at $S(850 \mu \mathrm{m})=2 \mathrm{mJy}$ is predicted to be 2.2 , significantly deeper than the prediction for $S(0.44 \mu \mathrm{m})=0.1 \mu \mathrm{Jy}(B=26.6)$. This shows the power of the $850 \mu \mathrm{m}$ surveys and also the difficulty there will be in identifying the sources detected.

Figure 10 shows the predicted integrated background spectrum for the 3 cosmological models. All are consistent with the data, though the predictions of the $\Omega_{o}=1$ model are on the low side, while those of the $\Lambda=0.7$ model are on the high side. Figure 10 (right) shows, for the $\Omega_{o}=1$ model, the contribution of the different SED types to the background. The dominant contribution is from the cirrus component at most wavelengths, so the prediction is that more of the energy from starbursts is deposited in the general interstellar medium of a galaxy than is absorbed in the early stages close to the location of the massive stars. This dominance by the cirrus component at submillimetre wavelengths also implies that many of the detected sources should turn out to be rather extended (kiloparsec scales rather than the more compact scales expected for nuclear starbursts). 

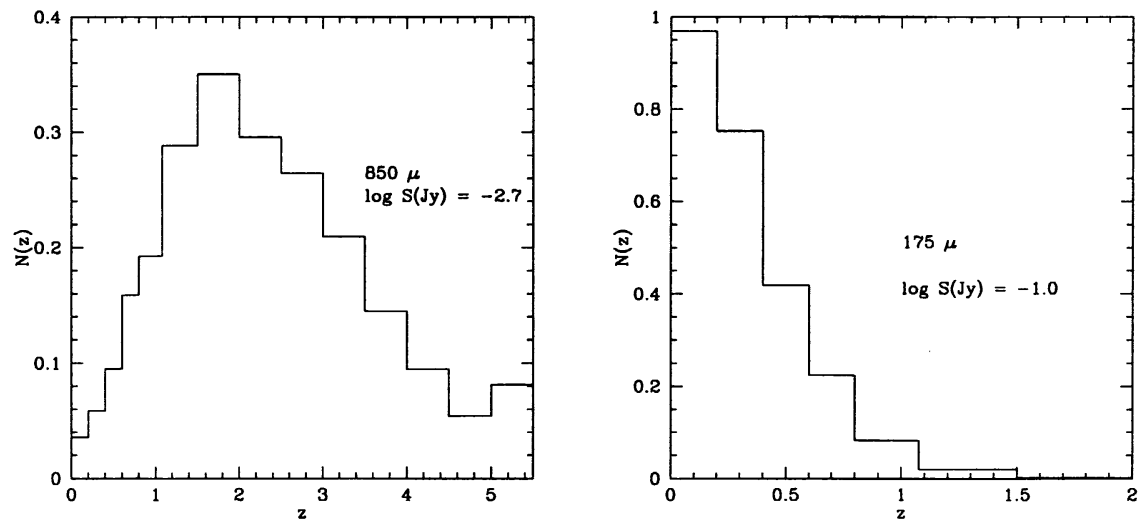

Figure 9. (Left) Redshift distribution at $850 \mu \mathrm{m}, \log _{10} S(\mathrm{Jy})=-2.7$. The bin centred at $z=5.25$ refers to $z>5$.

(Right) Redshift distribution at $175 \mu \mathrm{m}, \log _{10} S(\mathrm{Jy})=-1.0$.
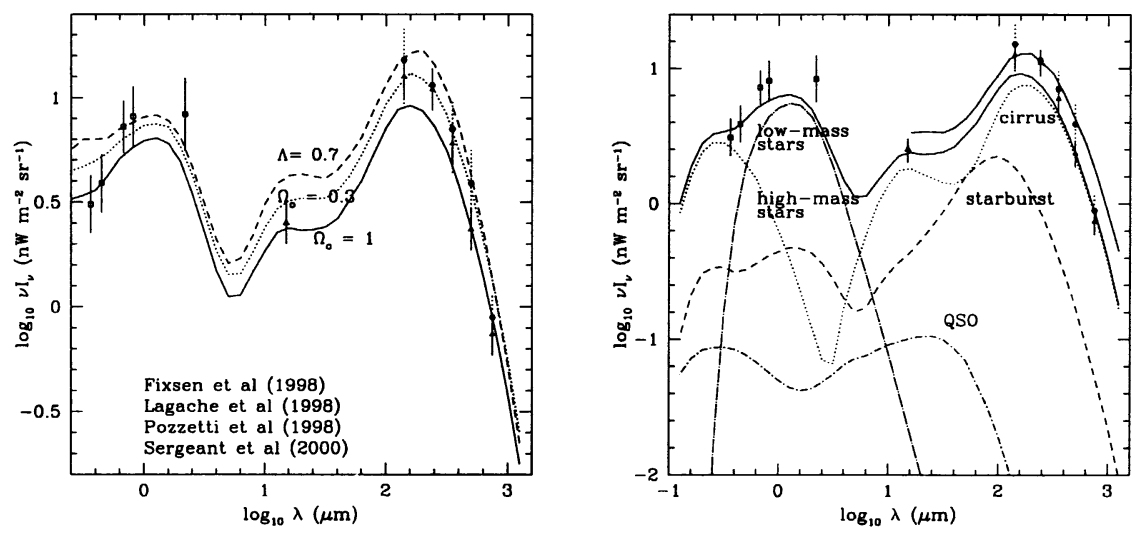

Figure 10. (Left) Predicted spectrum of integrated background for the same models as Fig. 5.

(Right) Predicted spectrum of integrated background for the $\Omega_{o}=$ 1 model, showing the contribution of the different components. The contribution of the Arp 220-like starbursts is less than $0.01 \mathrm{nW} \mathrm{m}^{-2}$ at all wavelengths. The upper solid curve at long wavelengths shows the effect of including density evolution (see text). Data from Fixsen et al. (1998) (far IR, submillimetre), Pozzetti et al. (1998) (optical, UV). 


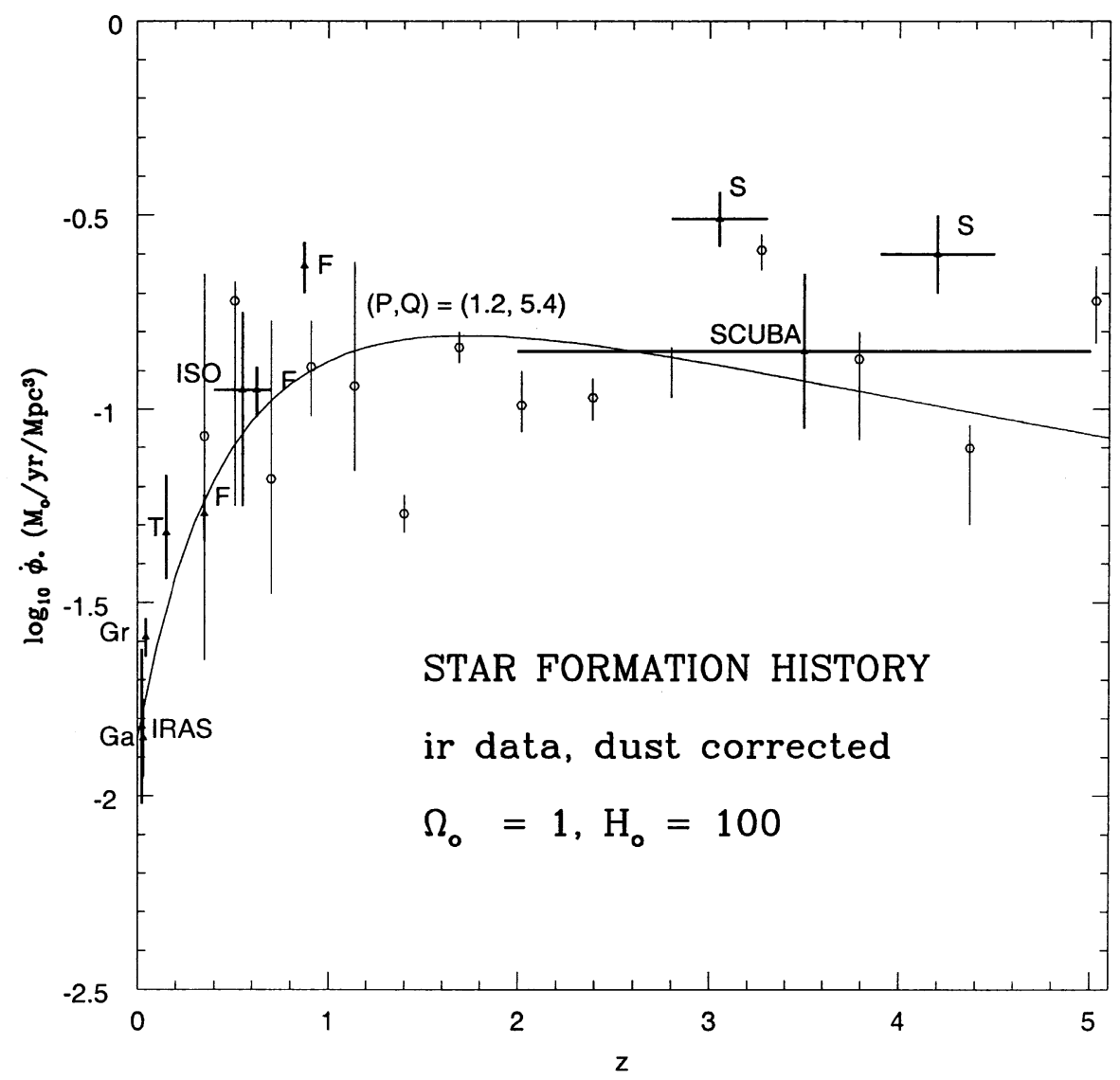

Figure 11. Direct estimates of the star formation rate, for $\Omega_{o}=1$, from infrared, submillimetre or radio data, or with correction for the effects of insterstellar dust, as a function of $z$. Data from Gallego et al. (1995), Rowan-Robinson (1997, revised: ISO), Gronwall (1998), Hughes et al. (1998, revised: SCUBA), Treyer et al. (1998), Flores et al. (1999), Steidel et al. (1999), and from photometric redshifts in the HDF (open circles, Rowan-Robinson 2000, in preparation). The ISO and SCUBA HDF points have been recalculated using a directly determined luminosity function (cf. Fig. 3, right-hand panel). The solid curve is the best-fitting model to the counts and background spectrum. 


\section{Effect of Including Density Evolution}

In the framework of hierarchical, bottom-up galaxy formation scenarios, like those based on cold dark matter, we expect that galaxies form as the result of mergers between smaller fragments. Thus, we might expect to see a higher total density of galaxies as we look back towards the past. To test whether attributing part of the star formation history to density evolution has a major effect on the predicted counts and backgrounds, I have considered, for the $\Omega_{o}=1$ case, a simple modification in which the comoving density of galaxies varies with redshift as

$$
\rho(z)=\rho(0)(1+z)^{n} .
$$

For $n=1$, this means that the comoving number-density of galaxies at redshifts 2,5 and 10 (our assumed cutoff redshift), is increased by a factor 3,6 and 11 compared with the present. This would represent a very substantial degree of merging of galaxies over the observable range of redshifts.

Since the background intensity depends on the product of the luminosity and density evolution rates, the background spectrum will be unaltered if we simply increase $P$ by $2 n / 3$. At low redshifts the counts will be unaffected if $P$ is increased by $n / 3$. I find that for a combined density evolution model with $n=1$ and luminosity evolution with $P=1.5, Q=5.4$ (and $\Omega_{o}=1$ ), the fits to the counts at $15-850 \mu \mathrm{m}$ hardly change over the observed range, but the predicted background is raised by about 0.2 dex, giving better agreement with observations for this cosmological model. For the $\Lambda=0.7$ model, the fit to the background would be significantly worsened for models consistent with the observed counts.

\section{Discussion and Conclusions}

I have developed a parametrized approach to the star formation history, which is sufficiently versatile to reproduce many proposed model histories. The model assumes that the evolution of the star formation rate manifests itself as pure luminosity evolution. I have stressed the importance of ensuring that the assumed luminosity function is consistent with available $60 \mu \mathrm{m}$ redshift survey data and that the assumed spectral energy distributions are realistic. The observed far IR and submillimetre counts and background then provide strong constraints on the model parameters.

The models consistent with infrared and submillimetre counts and backgrounds tend to show a flat star formation rate from $z=1-3$, consistent with the observed star formation history derived from HDF galaxies and other data (Fig. 11). The most striking difference from previous modelling work is the dominant role of the cirrus component at submillimetre wavelengths.

Areas requiring further work are (i) the need to consider the evolution of the shape of the SEDs, particularly for the cirrus component, with redshift. The increased star-formation rate at earlier times would tend to make the dust temperature higher, but this is partially offset by the lower abundance of low-mass stars at earlier times; (ii) the AGN dust tori models require a further parameter, the orientation, and this may affect the AGN counts at optical wavelengths; (iii) 
the tendency for the probability of finding an AGN component to increase with far infrared luminosity is not fully reflected in the approach followed here.

\section{References}

Barger, A. J., Cowie, L. L., \& Sanders, D. B., 1999, ApJ, 518, L5

Bruzual, A. G., \& Charlot, S. 1993, ApJ, 405, 538

Blain, A. W., et al. 1999a, MNRAS, 302, 632

Blain, A. W., et al. 1999b, ApJ, 512, L87

Blain, A., et al. 1999c, MNRAS, 309, 715

Boyle, B. J., Shanks T., \& Peterson, B. A. 1988, MNRAS, 235, 935

Dole, H., et al. 2000, in ESA SP-427, The Universe as seen by ISO, ed. P. Cox \& M. F. Kessler (Paris: ESA Special Publications), 1031, astro-ph/0002283

Dunne, L., et al. 2000, MNRAS, in press, astro-ph/0002234

Dwek, E., et al. 1998, ApJ, 508, 106

Eales, S., et al. 1999, ApJ, 515, 518

Efstathiou, A., Rowan-Robinson, M., \& Siebenmorgen, R. 2000, MNRAS, 313, 734

Elbaz, D., et al. 2000, A\&A, in press, astro-ph/9910406

Fixsen, D. J., et al. 1998, ApJ, 508, 123

Flores, H., et al. 1999, ApJ, 517, 408

Fox, M. J., et al. 2000, MNRAS, submitted

Franceschini, A., et al. 1997, in The Far IR and Submillimetre Universe, ESA SP-401, 159

Gregorich, D. T., et al. 1995, AJ, 110, 259

Guiderdoni, B., et al. 1998, MNRAS, 295, 877

Hacking, P. B., \& Houck, J. 1987, ApJS, 63, 311

Hauser, M. G., et al. 1998, ApJ, 508, 25

Hughes, D. H., et al. 1998, Nature, 394, 241

Kawara, K., et al. 1998, A\&A, in press

Lanzetta, K. M., Yahil, A., \& Fernandez-Soto, A. 1996, Nature, 381, 759

Lilly, S. J., et al. 1996, ApJ, 460, L1

Lonsdale, C. J., et al. 1990, ApJ, 358, 60

Loveday, J., et al. 1992, ApJ, 390, 338

McCracken, H. J., et al. 2000, MNRAS, in press, astro-ph/9904014

Madau, P., et al. 1996, MNRAS, 283, 1388

Metcalfe, N., Shanks, T., Fong R., \& Roche N. 1995, MNRAS, 273, 257

Meurer, G. R., et al. 1997, AJ, 114, 54

Meurer, G. R., Heckman, T. M., \& Calzetti, D. ApJ, 1999, 521, 64

Oliver, S., et al. 1997, MNRAS, 289, 471

Pearson, C., \& Rowan-Robinson, M. 1996, MNRAS, 283, 174

Pettini, M., et al. 1998, ApJ, 508, 539 
Pozzetti, L., et al. 1998, MNRAS, 298, 1133

Puget, J.-L., et al. 1996, A\&A, 308, 5

Rigopoulou, D., Lawrence, A., \& Rowan-Robinson M. 1996, MNRAS, 288, 1049

Rowan-Robinson, M. 1995, MNRAS, 272, 737

Rowan-Robinson, M., et al. 1997, MNRAS, 289, 490

Rush, B., Malkan, M. A., \& Spinoglio, L., 1993, ApJS, 89, 1

Saunders, W., et al. 1990, MNRAS, 242, 318

Serjeant, S., et al. 2000, MNRAS, 316, 738

Smail, I., Ivison, R. J., \& Blain, A. W. 1997, ApJ, 490, L5

Steidel, C. C., et al. 1999, ApJ, 519, 1

Verma, A., 2000, $\mathrm{PhD}$ thesis (Univ. of London)

Walker, T. P., et al. 1991, ApJ, 376, 51

$\mathrm{Xu}, \mathrm{C}$., et al. 1998, ApJ, 508, 576

\section{Discussion}

Martin Harwit: The star formation rate at high red shifts should be related to the heavy element production rate, since AGNs don't seem to be major contributors to the IR background. It will be interesting to see how abundance measurements affect current star formation models.

Michael Rowan-Robinson: At present the models do not allow for any variation of metallicity or dust properties with redshift. We might expect that the dust opacity was lower, and that the dust temperature was higher, at higher redshift. At long wavelengths, where there is greatest sensitivity to high redshifts, these two effects may partly cancel out.

Catherine Cesarsky: Did you attempt to fit the differential counts as well?

Rowan-Robinson: I haven't yet, but I plan to. I think the conclusion will be similar.

Eli Dwek: Can you comment on the different ranges of redshifts where the cirrus-dominated galaxies and starburts dominate the contribution to the observed cosmic infrared background $(\lambda \geq 140 \mu \mathrm{m})$ ?

Rowan-Robinson: At long wavelengths, my models always show the cirrusdominated galaxies dominating the counts and background.

Bruce Partridge: I'd like to urge the importance of radio observations in determining the star formation history (see Haarsma, D. B., et al., 2000, ApJ, 544,641 ). Radio observations are free of dust obscuration and provide generally secure optical identifications (and hence redshifts). Radio observations may prove useful in deciding between models like yours and those of Puget and others (and I'd urge you to include radio counts and redshift distributions in your models).

Rowan-Robinson: I agree that radio estimates provide an interesting check on star formation histories. I plan to extend the model to radio wavelengths shortly. 